\title{
Profile of darunavir in the management of treatment-experienced HIV patients
}

This article was published in the following Dove Press journal:

HIVIAIDS - Research and Palliative Care

25 September 2009

Number of times this article has been viewed

\section{Cameron Wolfe \\ Charles Hicks}

Duke University Medical Center, Division of Infectious Diseases, Durham, NC, USA
Correspondence: Cameron Wolfe

Duke University Medical Center, Division of Infectious Diseases, Box 3824, Durham, NC, 277I0, USA

Email cameron.wolfe@duke.edu
Abstract: Darunavir (formerly TMC114) is a second-generation, sulfonamide-based, peptidomimetic protease inhibitor (PI) with a modified 3-dimensional structure enabling more efficient binding to HIV protease. It has become an important drug, in combination with low-dose ritonavir boosting, in the treatment of both antiretroviral-naïve and multiclass-experienced patients. Growing data now exist suggesting it possesses a high barrier to resistance and requires multiple PI mutations in order to suffer reduced virological potency.

Keywords: darunavir, HIV, ritonavir

Since the approval of saquinavir in 1995, the protease inhibitor (PI) class has served an important role in constructing an effective highly active antiretroviral combination for patients with HIV. ${ }^{1}$ The PIs have several advantages over alternatives: they generally require greater numbers of resistance mutations to occur before they are rendered virologically ineffective (a property referred to as having a higher genetic barrier to resistance), and have not had the mitochondrial toxicities seen with some other agents. They have limitations, however, both in terms of their side effect profiles, and the cross resistance often inferred when PI mutations accumulate. ${ }^{2,3}$

Darunavir (DRV) represents a specifically engineered inhibitor of HIV-protease, designed to have a greater binding affinity within the active site of the viral enzyme. ${ }^{4}$ DRV requires co-administration with low-dose ritonavir (RTV) to achieve sufficiently concentrations for anti-HIV activity. On the basis of favorable results from a number of phase IIb clinical trials (described below), the US FDA granted 'accelerated approval' for DRV in June 2006, followed shortly after by European authorities in February 2007. It was subsequently approved for use in HIV-infected, antiretroviralnaïve patients.

\section{Structural determination of substrate binding to HIV-protease}

During viral maturation, HIV protease acts to cleave the post-translational proteins Gag and Gag-Pol to generate additional functional enzymes and structural proteins. ${ }^{4}$ This process is essential to achieve successful viral replication, thereby making protease an excellent target for antiretroviral agents. All approved PIs are competitive active-site inhibitors, and as such, would ideally mimic the enzyme substrate ensuring it interacts with the same residues on protease. ${ }^{5}$ 
Although structurally similar to amprenavir (see Figure 1), DRV has significantly greater binding avidity $\left(\mathrm{K}_{\mathrm{d}}=4.5 \times 10^{-12} \mathrm{M}\right)$, binding over 100 times tighter, and over 1000 times tighter than ritonavir, saquinavir or nelfinavir. ${ }^{6}$ The uniquely strong binding, and concurrent slow dissociation, are in part due to a complex of hydrogen bonds between a side chain on DRV and protease, similar to that found on native substrates. To illustrate this point, the mutation I84V is one of the most common PI mutations affecting the class as a whole. Although DRV binding affinity was affected in mutants harboring I84V, it was still at least 1.5 times stronger than all previous PI medications. Additionally, there is a second binding site on the protease surface of a V32I drugresistant mutant that confers additional activity for DRV, in contrast to older agents. ${ }^{7}$ The second site is on the surface of a flexible flap in the protease dimer. DRV also has some molecular flexibility, and can therefore adapt to varying shapes presented by mutant HIV-protease. ${ }^{8}$

\section{In vitro efficacy}

Preclinical studies revealed DRV to be a highly potent against HIV replication, in both wild-type and resistant viruses. DRV has a potent $50 \%$ effective concentration $\left(\mathrm{EC}_{50}\right)$ of 1 to $5 \mathrm{nM}$, and an $\mathrm{EC}_{90}$ of 2.7 to $13 \mathrm{nM}$, considerably lower than typical human concentrations, whereas there is no demonstrable cytotoxicty at $100 \mu \mathrm{M}$ (selectivity $>20,000$ ). ${ }^{9}$ Additionally, 50\% inhibitory concentration data $\left(\mathrm{IC}_{50}\right)$ suggests far greater efficacy compared to previous PIs: DRV IC $\mathrm{I}_{50}=0.003 \mu \mathrm{M}$, compared to a range of 0.017 to $0.047 \mu \mathrm{M}$ for saquinavir, amprenavir, indinavir, lopinavir, and ritonavir. ${ }^{10}$ Efficacy appears similar between HIV group M, group O, and recombinant forms. DRV also has potent activity in vitro against HIV-2. ${ }^{10}$

\section{Pharmacokinetic profile}

Darunavir has relatively poor bioavailability, metabolized primarily by the hepatic cytochrome P450 enzyme CYP3A4. Although there is a significant first pass effect, 'boosting' doses of RTV are used clinically to increase bioavailability from $37 \%$ to $82 \%$, and thus extend the elimination half-life to almost 15 hours. DRV concentrations in blood are increased by $30 \%$ when given with food, although the fat content of the meal does not seem to affect final concentrations. ${ }^{11}$ It is $95 \%$ protein bound in plasma, primarily to the alpha 1-acid glycoprotein, and achieves peak plasma concentration $\left(\mathrm{C}_{\max }\right)$ in roughly 3 hours. ${ }^{12}$

Dose-ranging studies indicated that DRV $600 \mathrm{mg}$, given with $100 \mathrm{mg}$ RTV, both twice daily, was the best combination of antiviral activity and diminished toxicity. It was selected for further study and became the initial licensed dose.

DRV is also distributed to compartments other than blood, achieving CSF concentrations sufficient to inihibit viral replication. ${ }^{13}$ Achievable drug levels in genital secretions are currently being assessed.

\section{Important clinical studies}

The first proof-of-concept trial for DRV was a 14-day multicenter trial of 3 boosted doses of DRV/ritonavir (DRV/r) (300/100 mg twice daily, 900/100 mg daily, and 600/100 mg twice daily) compared to a standard optimized background regimen. ${ }^{14}$ In the 50 patients studied, an HIV viral load less than 400 copies $/ \mathrm{mL}$ was achieved in $46 \%, 31 \%, 42 \%$, and $8 \%$ of patients, respectively, along with CD 4 count improvements of $16,5,63$, and 0.5 cells $/ \mu \mathrm{L}$. No DRV resistance was identified during this brief period.

\section{POWER I and 2 trials}

Initial phase IIb clinical trials assessed the activity of darunavir given with an optimized background regimen in HIV-infected patients with extensive prior antiretroviral treatment and multiclass resistance. POWER 1 (TMC114-C213) and POWER 2 (TMC114-C202) were both multinational partially blinded randomized controlled salvage trials. Inclusion criteria were similar across the two studies - treatment-experienced adults with an HIV viral load $>1000$ copies $/ \mathrm{mL}$, and at least one primary PI mutation. Both studies were conducted simultaneously in
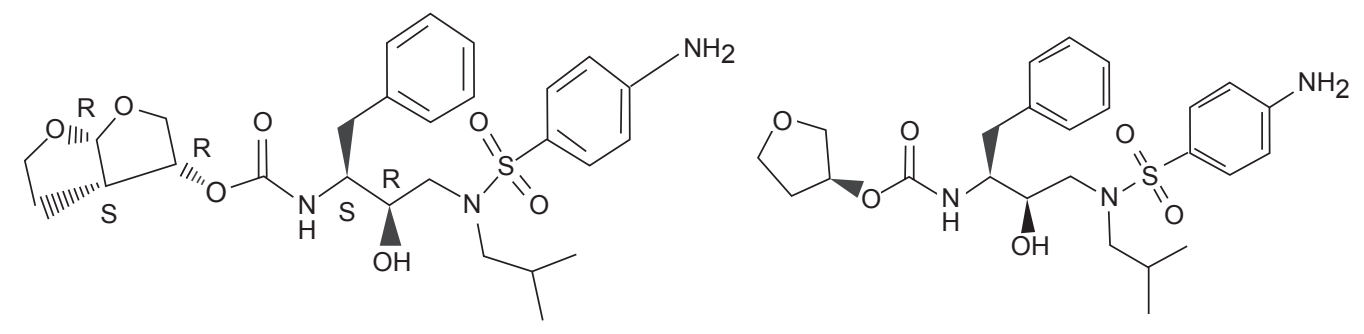

Figure I The structural similarity of darunavir (left) and amprenavir (right). 
different geographical areas, and contained 2 parts - an initial phase II dose-finding phase followed by a long-term phase in which participants either received boosted DRV or an investigator-selected comparator protease inhibitor. The primary efficacy endpoints in the POWER 1 and POWER 2 trials were the proportions of patients achieving a $\geq 1 \log _{10}$ reduction from baseline viral load. Secondary endpoints included changes from baseline CD4 count and the proportion of patients who achieving an undetectable HIV viral load (VL $<50$ copies/mL). Mean VL were 4.66 (DRV) and $4.48 \log _{10} \mathrm{c} / \mathrm{mL}$ (comparator) respectively, and baseline CD4 counts were 106 and 179 cells/ $\mu \mathrm{L} .{ }^{15}$ The interim analysis conducted at 24 weeks led to the eventual selection of DRV/r 600/100 mg twice daily as the dose selected for further clinical evaluation.

At closure (144 weeks), combined analysis of POWER 1 and 2 confirmed the prolonged activity of DRV in HIVinfected treatment-experienced patients, $51 \%$ of DRV recipients sustaining virologic suppression of $\geq 1 \log _{10}$ $(\mathrm{n}=131)$ compared to $10 \%$ of those on an optimized background therapy (OBT) $(n=124)$. Thirty-seven percent of those on DRV achieved a viral load less than 50 copies $/ \mathrm{mL}$, compared to $9 \%$ of those receiving OBT. Immunologically, the treatment group achieved a 97 cell $/ \mathrm{mL}$ increase compared to a $4 \mathrm{cell} / \mathrm{mL}$ increase (last observation carried forward analysis). ${ }^{16}$ Of note, at the time of the study tipranavir, raltegravir, maraviroc, and etravirine were not available. (enfuvirtide [T-20] was available at the investigator's discretion).

The use of enfuvirtide was associated with higher rates of viral suppression reflective of the importance of using more than one active agent in DRV regimens. With enfuvirtide first-time use, pooled data showed $56 \%$ of those in the DRV arm achieved a viral load $<50$ copies/mL at 48 weeks, versus $45 \%$ with no enfuvirtide. Without enfuvirtide this was still a satisfactory but clearly reduced $45 \%$. Importantly, if only those patients with no pre-existing DRV-associated resistance mutations (DRV-RAMS) were studied, the addition of enfuvirtide made no difference. Additionally, there is likely a selection bias among those given enfuvirtide, as evidenced by the fact that enfuvirtide recipients had a lower CD4 count at baseline with fewer active NRTIs in their background. Thus a true comparison is difficult to make. ${ }^{17}$

\section{POWER 3}

POWER-3 (TMC-114-C215) was an open-label safety and efficacy study in 327 patients, intended to expand the population of DRV patients for adverse events assessment.
In this similarly Caucasian male-dominated study, with similar inclusion criteria as POWER 1 and 2, mean baseline $\log _{10}$ HIV RNA $=4.58$ copies $/ \mu \mathrm{L}$, median CD4 cell count $=120$ cells $/ \mathrm{mm}^{3}, \mathrm{CDC}$ category $\mathrm{C}=55 \%$, and median number of primary PI mutations $=4 .{ }^{18,19}$ Using a TLOVR algorithm, 32\% achieved a viral load of $\leq 50$ copies $/ \mathrm{mL}$ at week 144 .

\section{TITAN}

The first direct head-to-head comparison of PI efficacy involving darunavir came with the phase III TITAN trial (TMC144-C214). TITAN was an open-label study comparing lopinavir/ritonavir (LPV/r) with DRV/r (600/100 mg twice daily) in patients with moderate previous PI experience. ${ }^{20}$ Geometric mean fold change (FC) was similar in both groups at randomization, although 58 patients had a $\mathrm{LPV} \mathrm{FC}>10$, compared to just 9 with DRV. There were also more patients in the DRV group with $\geq 2$ sensitive backbone ARVs used (non-PI), 203 (73\%) compared to 181 (63\%). Whether this affected the conclusion is unclear. After 96 weeks of treatment DRV was noninferior to LPV, the primary end-point of the trial (DRV $=67.5 \%$ to $\mathrm{LPV}=59.5 \%$ achieving VL $<400$ copies $/$ $\mathrm{mL}, P<0.001)$. In fact, DRV was superior to LPV based on a pre-defined end-point analysis $(P=0.034)$. Rates of virologic failure, as assessed by inability to suppress $\mathrm{VL}<400$ copies/mL by week 16 or viral rebound above 400 copies $/ \mathrm{mL}$ after initial suppression, was greater in the LPV arm, $25.6 \%$ vs $13.8 \%$. Data from the TITAN and the POWER studies led to accelerated approval of darunavir for use in treatment-experienced patients.

\section{ARTEMIS}

Based on observed response rates to DRV in patients with moderate prior PI exposure in the TITAN trial, the natural extension was to test efficacy in treatment-naïve patients. The ARTEMIS trial was a phase III open-label trial randomizing people to DRV/r 800/100 mg once daily or LPV/r 800/200 mg daily dose (given either twice daily [bid] or daily [qd]). 689 patients were randomized; trial participants began treatment with an average VL of about $4.85 \log _{10}(70,000$ copies) and a median CD4 count of 228 in the DRV group and 218 in the lopinavir (LPR) group. About 60\% in each treatment arm had subtype B virus, and fewer than $10 \%$ in each arm had AIDS. At 48 weeks DRV/r was not inferior to $\mathrm{LPR} / \mathrm{r}$, with $84 \%$ of the DRV/r group achieving a VL $<50$ copies, compared to $78 \%$ in the LPV group. Response rates for those with baseline $\mathrm{VL} \geq 100,000$ were better in the DRV $\operatorname{arm}(79 \%$ vs $67 \%$ response, $P<0.05)$, as were responses in 
those with baseline CD4 count $<200$ cells/ $\mu \mathrm{L}$. DRV efficacy seemed to be independent of any individual variation in pharmacokinetics. ${ }^{21}$

Longer term follow-up (through to 96 weeks) confirmed the initial results: $79 \%$ of DRV patients had achieved a $\mathrm{VL}<50$ copies/mL compared to $71 \%$ receiving LPV (difference $=8.3 \%, P$ value for superiority $=0.012$, ITT-TLOVR). ${ }^{22}$ Interestingly suboptimal adherence (based on self-reporting using a validated questionnaire) appeared to affect DRV responses less than LPV response (7\% vs $25 \%$ difference compared to adherent patients, respectively).

\section{Other studies}

In pediatric populations, DRV/r has similar efficacy. The DELPHI study (TMC114-C212) was an open-label phase II study of treatment-experienced HIV-1-infected patients between the ages of 6 and 17 years. Dosing was weightbased: either $375 / 50 \mathrm{mg}$ bid (44 to $66 \mathrm{lbs}$ [20 to $30 \mathrm{~kg}$ ), $450 / 60 \mathrm{mg}$ bid (66 to $88 \mathrm{lbs}$ [20 to $40 \mathrm{~kg}$ ) or $600 / 100 \mathrm{mg}$ bid (weight $>88 \mathrm{lbs}$ [40 kg]). Mean age was 14 years, mean CD4 count was 330 cell $/ \mu \mathrm{L}$ and mean $\mathrm{VL}=4.64 \log _{10}$. At 24 weeks $\mathrm{VL}<50$ copies was achieved in $50 \%$ of the population, and less than 400 copies in $64 \%$. Average CD4 count increase was 117 cells $/ \mu \mathrm{L}$. Pharmacokinetic results were similar to adults, and the drug was generally well tolerated. ${ }^{23} \mathrm{DRV}$ was approved for use in pediatric populations in December 2008 in the US.

Additional research will illuminate treatment responses in other populations. The GRACE trial (TMC114HIV3004) is focused on DRV responses among women and ethnic minorities. The ODIN trial (TMC114-C229) hopes to expand on preliminary information suggesting 800/100 mg DRV/r given once daily is effective even in treatment-experienced patients provided they have no specific DRV mutations. Pilot studies have already been completed ${ }^{24}$ and subanalysis of POWER data suggests the approach worthy. ${ }^{25}$ Currently, following the TITAN and POWER studies the 600/100 mg bid dosing is still recommended. In line with similar studies from older PIs, the MONET trial is taking patients with well controlled HIV (VL $<50$ copies/mL for at least 24 weeks and randomizing them to receive triple-drug therapy including DRV, or 800/100 mg DRV/r monotherapy.

\section{Drug resistance}

Initial in vitro viral passage studies suggested mutants showing resistance to darunavir evolved slowly, ${ }^{9}$ and were difficult to predict. Subsequent clinical trials have attempted to quantify which preexisting mutations were likely to deleteriously impact on darunavir efficacy. Data from the POWER studies and the DUET trials (of etravirine) helped generate a list of DRV-RAMS. The International AIDS Society (IAS-USA) currently lists 11 major mutations for darunavir: V11I, V32I, L33F, I47V, I50V, I54L/M, T74P, L76V, I84V, and L89V. Of these I50V, I54M/L and I84V have been identified as major mutations, referring to their tendency to be selected earlier in the presence of DRV or substantial reduction of DRV susceptibility. Mutations in the protease flap region are recognized as critical for modified binding of a number of PIs. This explains, for example, the observed clinical darunavir resistance noted with $150 \mathrm{~V}$ which forms part of this region. ${ }^{26}$ Interestingly, when many of the other DRV-RAMs were included in a wild-type genetic background by site-directed mutagenesis, they did not cause decreased susceptibility to DRV, suggesting numerous additional PI mutations were required for resistance. ${ }^{27}$

There have been suggestions that some patients harboring the amprenavir-specific resistance profiles, such as I50V or V32I + I47V, failed on a DRV/r-containing therapy. ${ }^{28}$ Larger analysis of the POWER studies showed among patients with a high level of phenotypic resistance to (fos)amprenavir (FC > 11.4), response to DRV/r was lower than those with a high level of phenotypic resistance (31\% to $66 \%$ at 48 weeks). Despite this there was no association with previous (fos)amprenavir use and DRV failure per se. ${ }^{29}$

The number of baseline primary PI mutations, as identified by the IAS-USA had minimal effect on virologic outcomes, as patients with 0 mutations in the TITAN trial achieved suppression in $84 \%(139 / 165)$ as did patients with $\geq 3$ baseline mutations. ${ }^{20}$ There does, however, appear to be a strong correlation with the number of DRV-specific RAMs, $\geq 3$ being associated with a DRV FC $>10$, and therefore a less favorable outcome. ${ }^{17}$ When stratified according to phenotypic FC, an $\mathrm{FC}<10$ from baseline resulted in viral load reduction of $2.08 \log _{10}$ copies $/ \mathrm{mL}$, an $\mathrm{FC}=10$ to 40 resulted in decrease of $1.08 \log _{10}$ copies $/ \mathrm{mL}$, and an FC $>40$ resulted in only a $0.76 \log _{10}$ copies $/ \mathrm{mL}$. Proportions of patients achieving an undetectable VL were $50 \%, 25 \%$, and just $13 \%$ respectively. Within the POWER studies, the proportion of patients achieving a RNA VL $<50$ copies $/ \mathrm{mL}$ declined as the number of RAMs increased: $60 \%$ with zero RAMs, $45 \%$ with 1 to 2 and $\leq 20 \%$ with 3 or more.

More recently, a number of other mutations have been detected, following a multivariate analyses of 153 French patients receiving salvage DRV/r, although these have not been added to the IAS-USA DRV RAM list. ${ }^{30}$ 
As clinicians begin to use DRV/r earlier in treatment of HIV-infected patients, concerns remain as to future PI susceptibility in those experiencing virologic failure. Trial data of those failing initial DRV-containing regimens suggest few new resistance mutations develop. By week 48 in ARTEMIS, patients experiencing viral rebound had not developed any IAS-USA PI resistance mutations, ${ }^{31}$ although one NRTI mutation was noted, M184V. In the POWER studies, those with virologic failure were noted to have a small number of mutations already recognized as DRV-RAMs (V32I, L33F, I47V, I54L, and L89V). Similar results were seen in the French cohort with the addition of V11I, and $\mathrm{I} 50 \mathrm{~V}$, also both contained within the most recent IAS-USA list of DRV mutations. ${ }^{32}$

The sparsity of mutagenesis in those failing DRVcontaining regimens suggests that, as with other PIs, virologic failure tends to be associated with poor adherence. In the absence of preexistent DRV resistance, newly acquired DRV-RAMs were also rare. Certainly DRV/r appeared to spare future PI use more so than $\mathrm{LPV} / \mathrm{r}$ in the TITAN trial, as seen in Figure 2.

Baseline tipranavir susceptibility was maintained in a number of studies, despite DRV virological failure, suggesting it will remain an option, even in the heavily treatment-experienced population. ${ }^{32,33}$ This may possibly be because of the 21 recognized tipranivir resistance mutations, only 5 (V11I, V33F, I47V, I54M, and I84V) are also DRV-RAMs. ${ }^{8,34}$ Conversely $70 \%$ of isolates seen to have dimished tipranavir susceptibility still retained susceptibility to DRV. Further clinical data on the resistance development in the antiretroviral-naïve population using DRV will help verify the absence of resistance in most persons with virologic failure.

\section{Presentation}

DRV is packaged as a direct compression tablet, now produced in the US as either a $400 \mathrm{mg}$ or $600 \mathrm{mg}$ formulation (the $300 \mathrm{mg}$ dose is no longer available). A smaller $75 \mathrm{mg}$ tablet has been released to dovetail with the approval in pediatric populations. Dosing for adults should at present be $600 / 100 \mathrm{mg}$ bid with RTV for treatment-experienced adults, or $800 / 100 \mathrm{mg}$ daily for treatment-naïve adults. Pediatric doses vary with weight, as shown in Table 1. A liquid formulation is currently under investigation. Similar dosing is recommended for all patients irrespective of renal function, although data are limited for hemodialysis, hemofiltration, or peritoneal dialysis. Dosing guidelines for patients with severe hepatic impairment suggest caution.

\section{Safety}

In clinical trials DRV/r has been generally well tolerated. The most significant adverse event has been hepatotoxicity, such that the FDA released an official warning in March 2008. The incidence of significant hepatotoxicity is about $0.5 \%$ in clinical trials. Although the more common abnormalities have been acute hepatitis and cytolytic hepatitis, rare fulminant cases and a number of deaths have occurred, although generally in patients with advanced disease who were taking multiple concominant medications. A substudy of patients co-infected with hepatitis B and/or C within the POWER

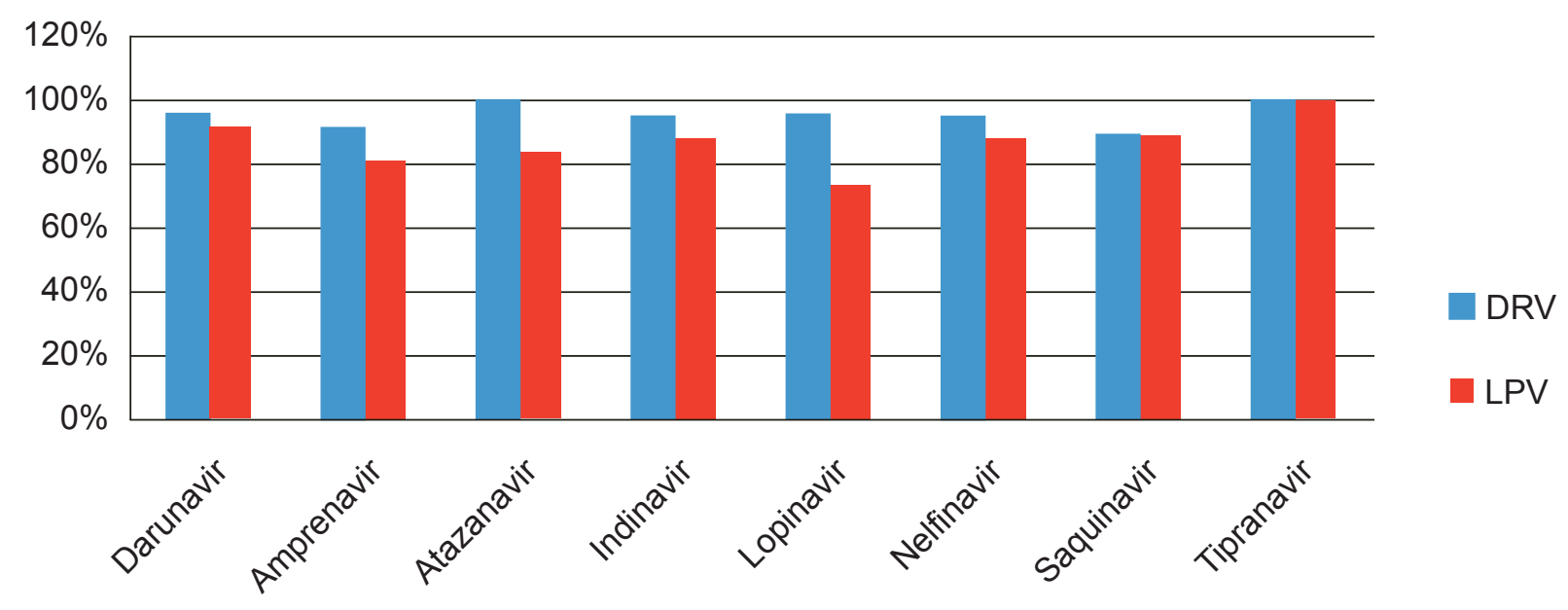

Figure 2 Percentage of patients in whom pretreatment isolates that were susceptible to protease inhibitors remained so after failure of darunavir/ritanovir (DRV/RTV) or lopinavir/ritanovir (LPV/RTV) in the TITAN trial. Drawn from data of de Meyer. ${ }^{35}$ 
Table I Recommended dose for pediatric patients (6 to $<18$ years of age) for Prezista ${ }^{\circledR}$ tablets with ritonavir body weight dose ${ }^{36}$

\begin{tabular}{|c|c|c|}
\hline \multicolumn{2}{|l|}{ Body weight } & \multirow[t]{2}{*}{ Dose } \\
\hline (kg) & (Ibs) & \\
\hline$\geq 20 \mathrm{~kg}$ to $<30 \mathrm{~kg}$ & $\geq 44$ lbs to $<66$ lbs & $\begin{array}{l}375 \mathrm{mg} \text { darunavir/50 mg } \\
\text { ritonavir twice daily }\end{array}$ \\
\hline$\geq 30 \mathrm{~kg}$ to $<40 \mathrm{~kg}$ & $\geq 66$ lbs to $<88 \mathrm{lbs}$ & $\begin{array}{l}450 \mathrm{mg} \text { darunavir } / 60 \mathrm{mg} \\
\text { ritonavir twice daily }\end{array}$ \\
\hline$\geq 40 \mathrm{~kg}$ & $\geq 88 \mathrm{lbs}$ & $\begin{array}{l}600 \mathrm{mg} \text { darunavir/100 mg } \\
\text { ritonavir twice daily }\end{array}$ \\
\hline
\end{tabular}

trials showed more coinfected patients to have liver-relatied adverse events compared to their mono-infected counterparts, although numbers were small. ${ }^{37}$ Rates were similar in those receiving DRV or the investigator selected PI. Whether adverse hepatic events occur more frequently in those with severe hepatic synthetic impairment or in those who develop immune reconstitution inflammatory syndrome remains to be determined. ${ }^{36}$

In the major DRV trials, rates of side effects were generally low. Rash is one of the most common (16\% to $7 \%$ compared with LPV in TITAN), most likely due to the sulfonamide moiety with DRV. Reassuringly $<1 \%$ of users developed a severe or life-threatening rash (Stevens-Johnson syndrome), with a $0.3 \%$ discontinuation rate due to rash. Only 7\% of patients receiving DRV discontinued for any reason. ${ }^{38}$ Gastrointestinal (GI) adverse events of any kind occur less than frequently than LPV (75\% vs $61 \%)$. In the POWER studies, the most common adverse events $(>10 \%$ regardless of severity or causality) were diarrhea, headache, nausea, and fatigue. ${ }^{15,16}$ Reported GI events occurred in 7\% of adherent patients compared to $28 \%$ of the suboptimally adherent.

Longer-term safety profiles of antiretroviral agents are increasingly important as patients stay on medications longer. Occasional lipodystrophy, hyperlipidemia. and worsening insulin resistance have been reported. Triglyceride levels and total cholesterol rose in the ARTEMIS cohort although less with DRV than with LPV, as may have been expected. Findings were equivalent for elevations in mean low-density lipoprotein and total cholesterol/high-density lipoprotein ratio. ${ }^{31}$ Small weight gains were seen in trial patients; however, there were no associated with changes in body shape and it was difficult to distinguish between change likely from general health restoration. ${ }^{31}$

There have been no adequate and well controlled studies in pregnant women. DRV therefore is currently classified as a Category $\mathrm{C}$ drug, because animal data in which there was no detected teratogenicity in mice, rabbits, and rats. It is passed into breast milk.

\section{Drug interactions}

Especially because DRV needs RTV boosting, there are a number of significant drug interactions to consider. These are generally applicable across the majority of the PIs. Rather than a comprehensive list, a selection of important or life-threatening interactions is presented in Appendix 1. ${ }^{36}$ One interaction that deserves special attention is that of $\mathrm{DRV} / \mathrm{r}$ with rifampin. Coadministration leads to a marked decrease in plasma DRV levels that in turn may lead to a loss of therapeutic effect. RTV does not overcome this interaction, and hence the combination is not recommended. Given the importance of the rifamicins in the treatment of Mycobacterium tuberculosis, substitution with rifabutin is usually recommended. DRV/r conversely increases the AUC and $\mathrm{C}_{\max }$ of rifabutin, so it should be dosed at $150 \mathrm{mg}$ every Monday/Wednesday/Friday.

\section{Clinical roles of darunavir}

As with most drugs approved in the last 5 years, DRV was first shown to be efficacious in highly treatment-experienced patients. Subsequently studies have shown efficacy with once daily dosing, including children and treatment-naïve patients. Its tolerability, dosing flexibility, high potency, and favorable resistance characteristics have made it an increasingly popular drug for HIV-infected person in all states of infection. As with all antiretroviral agents, DRV/r should be given with other fully active drugs based on resistance testing. Fortunately several new antiretroviral agents have become available recently. Raltegravir, the first HIV integrase inhibitor, seems to work well with DRV/r. In the BENCHMRK studies, approximately $60 \%$ of the patients received DRV/r as part of their optimized background, and subgroup analysis of those patients revealed especially high response rates. ${ }^{39,40}$

Similarly in the DUET trials of etravirine, DRV was frequently used as part of an optimized background. ${ }^{41}$ Combinations of newer antiretroviral agents have proven to be quite efficacious. In a small cohort of 103 French patients, with triple-class experience, the combination of DRV/r, etravirine and raltegravir did especially well. ${ }^{42}$ Fifty-five percent of this highly resistant group had an undetectable VL by week 4 , and $93 \%$ by week 24 had VL $<50$ copies $/ \mathrm{mL}$. CD4 lymphocytes increased to a mean of 99 cells $/ \mu \mathrm{L}$, and only 1 patient discontinued the regimen (due to rash). Further 'real world' clinical studies using combinations of 
the newer agents will be required, but for now, these results are encouraging.

In the antiretroviral-naïve patient, DRV/r 800/100 mg now offers an alternative as a first-line PI regimen. Advantages are a relatively low pill burden and an excellent profile for efficacy, resistance, and safety. Co-administration with ritonavir remains problematic with DRV as with other PIs, for many reasons - RTV for many is burdensome in terms of its capsule size, need for refrigeration, complex drug-drug interactions, and GI side effects.

\section{Conclusion}

Darunavir, a recently approved HIV protease inhibitor, offers clinicians an important option for the treatment of treatmentnaïve and -experienced patients. It has a favorable side effect profile, is accordingly well tolerated, and has shown excellent capacity to suppress HIV viral load and engender CD4 cell recovery. Importantly, resistance to darunavir is relatively uncommon, facilitating prolonged efficacy in patients.

\section{Disclosures}

Cameron Wolfe discloses no conflicts of interest.

Charles Hicks has received research support or honoraria from Abbott, Bristol-Myers Squibb, Glaxo SmithKline, Gilead, Merck, Pfizer, Tibotec, Kpronis, Myriad, and Schering Plough.

\section{References}

1. Moore RD, Chaisson RE. Natural history of HIV infection in the era of combination antiretroviral therapy. AIDS. 1999;13(14):1933-1942.

2. Hertogs K, et al. Phenotypic and genotypic analysis of clinical HIV-1 isolates reveals extensive protease inhibitor cross-resistance: a survey of over 6000 samples. AIDS. 2000;14(9):1203-1210.

3. van Roon EN, et al. Incidence of discontinuation of highly active antiretroviral combination therapy (HAART) and its determinants. J Acquir Immune Defic Syndr Hum Retrovirol. 1999;20(3):290-294.

4. Lefebvre E, Schiffer CA. Resilience to resistance of HIV-1 protease inhibitors: profile of darunavir. AIDS Rev. 2008;10(3):131-142.

5. Surleraux DL, et al. Discovery and selection of TMC114, a next generation HIV-1 protease inhibitor. J Med Chem. 2005;48(6):1813-1822.

6. King NM, et al. Structural and thermodynamic basis for the binding of TMC114, a next-generation human immunodeficiency virus type 1 protease inhibitor. J Virol. 2004;78(21):12012-12021.

7. Kovalevsky AY, et al. Solution kinetics measurements suggest HIV-1 protease has two binding sites for darunavir and amprenavir. $\mathrm{J} \mathrm{Med}$ Chem. 2008;51(20):6599-6603.

8. Tremblay CL. Combating HIV resistance - focus on darunavir. Ther Clin Risk Manag. 2008;4(4):759-766.

9. De Meyer S, et al. TMC114, a novel human immunodeficiency virus type 1 protease inhibitor active against protease inhibitor-resistant viruses, including a broad range of clinical isolates. Antimicrob Agents Chemother. 2005;49(6):2314-2321.

10. Koh Y, et al. Novel bis-tetrahydrofuranylurethane-containing nonpeptidic protease inhibitor (PI) UIC-94017 (TMC114) with potent activity against multi-PI-resistant human immunodeficiency virus in vitro. Antimicrob Agents Chemother. 2003;47(10):3123-3129.
11. Sekar V, et al. The effect of different meal types on the pharmacokinetics of darunavir (TMC114)/ritonavir in HIV-negative healthy volunteers. J Clin Pharmacol. 2007;47(4):479-484.

12. Rittweger M, Arasteh K. Clinical pharmacokinetics of darunavir. Clin Pharmacokinet. 2007;46(9):739-756.

13. Yilmaz A, et al. Darunavir concentrations in cerebrospinal fluid and blood in HIV-1-infected individuals. AIDS Res Hum Retroviruses. 2009;25(4):457-461.

14. Arasteh K, et al. TMC114/ritonavir substitution for protease inhibitor(s) in a non-suppressive antiretroviral regimen: a 14-day proof-of-principle trial. AIDS. 2005;19(9):943-947.

15. Katlama C, et al. Efficacy and safety of TMC114/ritonavir in treatmentexperienced HIV patients: 24-week results of POWER 1. AIDS. 2007;21(4):395-402.

16. Katlama C, et al. POWER 1 and 2: combined final 144-week efficacy and safety results for darunavir/ritonavir $600 / 100 \mathrm{mg}$ bid in treatmentexperienced HIV patients. 9th International Congress on Drug Therapy in HIV Infection; 2008. Glasgow, UK.

17. Gathe J. Examination of factors influencing response to darunavir combined with low-dose ritonavir in POWER 1, 2, and 3: Pooled 48-week analysis. Frontiers in Drug Development for Antiretroviral Therapies; 2006. Cancun, Mexico.

18. Pozniak A, et al. POWER 3 analysis: 144-Week efficacy and safety results for darunavir/ritonavir (DRV/R) 600/100 mg BID in treatmentexperienced HIV patients. Ninth International Congress on Drug Therapy in HIV Infection; 2008. Glasgow, UK.

19. Pozniak A, et al. Effect of baseline viral susceptibility on response to darunavir/ritonavir versus control protease inhibitors in treatmentexperienced HIV type 1-infected patients: POWER 1 and 2. AIDS Res Hum Retroviruses. 2008;24(10):1275-1280.

20. De Meyer $S$, et al. Influence of baseline protease inhibitor resistance on the efficacy of darunavir/ritonavir or lopinavir/ritonavir in the TITAN trial. J Acquir Immune Defic Syndr. 2008;49(5):563-564.

21. Sekar V, et al. Pharmacokinetic-pharmacodynamic analyses of once-daily darunavir in the ARTEMIS study. 15th Conference on Retroviruses and Opportunistic Infections; 2008. Boston, MA, USA.

22. Nelson M, et al. Adherence to darunavir/ritonavir and lopinavir/ritonavir in treatment-naive, HIV-infected patients in ARTEMIS: 96-week data. 15th Annual Conference of the British HIV Association (BHIVA); 2009. Liverpool, UK.

23. Bologna R, et al. Safety and efficacy of darunavir co-administered with low-dose ritonavir in treatment-experienced children and adolescents at Week 24. Fifteenth Conference on Retroviruses and Opportunistic Infections; 2008. Boston MA, USA.

24. Curran A, et al. Pharmacokinetics, efficacy and safety of darunavir/ ritonavir 900/100 mg once-daily. 10th international Workshop on Clinical Pharmacology of HIV Therapy; 2009. Amsterdam, NL.

25. De Meyer SM, et al. Efficacy of once-daily darunavir/ritonavir 800/100 mg in HIV-infected, treatment-experienced patients with no baseline resistance-associated mutations to darunavir. J Acquir Immune Defic Syndr. 2008;49(2):179-182.

26. Liu F, et al. Effect of flap mutations on structure of HIV-1 protease and inhibition by saquinavir and darunavir. J Mol Biol. 2008;381(1): $102-115$.

27. de Meyer S, et al. Resistance profile of darunavir: combined 24-week results from the POWER trials. AIDS Res Hum Retroviruses. 2008;24(3):379-388.

28. Delaugerre $\mathrm{C}$, et al. Key amprenavir resistance mutations counteract dramatic efficacy of darunavir in highly experienced patients. AIDS. 2007;21(9):1210-1213.

29. Picchio G, et al. Response to "key amprenavir resistance mutations counteract dramatic efficacy of darunavir in highly experienced patients". AIDS. 2008;22(1):165-167.

30. Descamps D, et al. Mutations associated with virological response to darunavir/ritonavir in HIV-1-infected protease inhibitorexperienced patients. J Antimicrob Chemother. 2009;63(3): $585-592$. 
31. Ortiz R, et al. Efficacy and safety of once-daily darunavir/ritonavir versus lopinavir/ritonavir in treatment-naive HIV-1-infected patients at week 48. AIDS. 2008;22(12):1389-1397.

32. Delaugerre $\mathrm{C}$, et al. Pattern and impact of emerging resistance mutations in treatment experienced patients failing darunavir-containing regimen. AIDS. 2008;22(14):1809-1813.

33. Daar ES. Emerging resistance profiles of newly approved antiretroviral drugs. Top HIV Med. 2008;16(4):110-116.

34. Johnson VA, et al. Update of the drug resistance mutations in HIV-1. Top HIV Med. 2008;16(5):138-145.

35. de Meyer S. Characterization of virologic failures on darunavir/ ritonavir in the randomized, controlled, Phase III TITAN trial in treatment-experienced patients. 15th Conference on Retroviruses and Opportunistic Infections; 2008. Boston, MA, USA.

36. Prezista ${ }^{\circledR}$ [package insert]. 2009. TibotecTherapeutics, East Bridgewater, NJ, USA.

37. Rachlis A, et al. Safety, tolerability, and efficacy of darunavir (TMC114) with low-dose ritonavir in treatment-experienced, hepatitis B or C co-infected patients in POWER 1 and 3. HIV Clin Trials. 2007;8(4):213-220.
38. Hirschel B, Perneger T. No patient left behind - better treatments for resistant HIV infection. Lancet. 2007;370(9581):3-5.

39. Cooper DA, et al. Subgroup and resistance analyses of raltegravir for resistant HIV-1 infection. N Engl J Med. 2008;359(4):355-365.

40. Steigbigel RT, et al. Raltegravir with optimized background therapy for resistant HIV-1 infection. N Engl J Med. 2008;359(4): 339-354.

41. Madruga JV, et al. Efficacy and safety of TMC125 (etravirine) in treatment-experienced HIV-1-infected patients in DUET-1: 24-week results from a randomised, double-blind, placebo-controlled trial. Lancet. 2007;370(9581):29-38.

42. Yazdanpanah Y, et al. High rate of virologic success with raltegravir plus etravirine and darunavir/ritonavir in treatment-experienced patients with multidrug-resistant virus: results of the ANRS 139 TRIO trial. XVII International AIDS Conference (AIDS 2008); 2009. Mexico City, Mexico.

43. Sekar VJ, et al. Pharmacokinetic interaction between ethinyl estradiol, norethindrone and darunavir with low-dose ritonavir in healthy women. Antivir Ther. 2008;13(4):563-569. 
Appendix I Interactions with darunavir/ritonavir ${ }^{36,43}$

\section{Drug class}

Anti-infectives

Cardiovascular medication

Immunosuppressants

Psychotropics

Erectile dysfunction

Illicit drugs

Herbals

Antiretrovirals

Contraceptives

Anticonvulsants

Drug of concern

rifampicin

rifabutin

voriconazole

fluconazole

itraconazole/ketaconazole

halofantrine/lumafantrine

amiodarone

lidocaine (lignocaine)

warfarin

$\mathrm{CCB}, \mathrm{BBs}$

statin class

cyclosporin/tacrolimus/sirolimus

SSRI

respiridone/clozapine/haloperidol

sildenafil/vardenafil

amphetamines, gamma-hydroxybutyrate

St John's wort

maraviroc

ethinylestradiol/norethindrone

phenytoin, phenobarbital

\section{Interaction}

Reduced DRV levels, avoid

Increased rifabutin levels and toxicity; recommended rifabutin dose reduction to $150 \mathrm{mg}$ every other day

Significant decrease in voriconazole level expected with $\mathrm{DRV} / \mathrm{r}$ - must use with caution and possible use therapeutic drug monitoring of voriconazole; individually, $39 \%$ reduction and $24 \%$ reduction in voriconazole dose with DRV and RTV respectively No significant interaction Increased azole and DRV levels

Avoid - increased risk of prolonged QTc via reduced P450-3A4 metabolism

Increased levels of amiodarone expected, watch for cardiac arrhythmia

Increased levels of lidocaine expected, watch for cardiac arrhythmia

Reduced warfarin exposure ( $21 \%$ following a single dose), should check INR levels more frequently

Potential increase in most $\mathrm{CCB}$ and BBs

Increased levels of most statins (reduced metabolism via 3A4); avoid simvastatin, caution and possible dose reduction with pravastatin/atorvastatin

Expected increased levels of immunosuppressant recommend therapeutic drug monitoring

Generally reduced SSRI levels, watch therapeutic response

Increased psychotropic levels, watch therapeutic response

Increased levels, suggest lower doses

Increased levels and possibly toxicity from illict agents Decreased DRV levels. Avoid. Prolonged effect even after discontinuation

Complex interaction - expected $4 \times$ increase in AUC and $2.3 \times$ increase in $C_{\max }$ of maraviroc, but recommend careful review of all medications Significant decreased levels - recommend seek alternative contraceptive methods

Decreased concentrations of anticonvulsants and DRV; avoid if possible

Abbreviations: AUC, area under the curve; $B B$, beta-blockers; $C_{\text {max }}$, maximum concentration; $C C B$, calcium-channel blockers; $D R V$, darunavir; INR, international normalized ratio; RTV, ritanovir; SSRI, selective serotonin reuptake inhibitors.

\section{Publish your work in this journal}

HIV/AIDS - Research and Palliative Care is an international, peerreviewed open-access journal focusing on advances in research in HIV, its clinical progression and management options including antiviral treatment, palliative care and public healthcare policies to control viral spread. The journal welcomes original research, basic science,

\section{Dovepress}

clinical \& epidemiological studies, reviews \& evaluations, expert opinion \& commentary, case reports \& extended reports. The manuscript management system is completely online and includes a very quick and fair peer-review system. Visit http://www.dovepress.com/ testimonials.php to read real quotes from published authors.

Submit your manuscript here: http://www.dovepress.com/hivaids---research-and-palliative-care-journal 